Results: Between September and October 2019, 434 participants completed the survey. Preliminary results show that most participants (78\%) contacted one or more health care professional(s) for their knee OA complaints in the last year; most often an exercise and/or physical therapist (53.2\%), the general practitioner $(43.8 \%)$ and/or an orthopedist/orthopedic surgeon (41.5\%). Furthermore, there were large variations in self-reported quality indictor achievements (checked "Yes") with the lowest rate for referral for weight reduction and, the highest rate for advice on managing/living with osteoarthritis (table 1). On average, a score of 6.5 was given by the participants for the quality of knee OA healthcare in the Netherlands. Participants' suggestions for OA care improvement comprised of, among others, more attention to pain and fatigue symptoms, expanding and researching treatment options, more information on OA, more attention to the personal situation and, improvement of multidisciplinary care.

Table 1. Self-reported Quality Indicator achievement

\begin{tabular}{lcc}
\hline Self-reported Indicators & Eligible $\mathrm{n}^{*}$ & $\begin{array}{c}\text { Checked } \\
\text { "Yes" } \mathrm{n}(\%)\end{array}$ \\
\hline Received information about OA & 417 & $310(74.3)$ \\
Received Information about treatment & 424 & $252(59.4)$ \\
Advised on managing/living with OA & 421 & $313(74.4)$ \\
Was offered support on managing/living with OA & 421 & $253(60.1)$ \\
Received information about exercise & 426 & $306(71.8)$ \\
Was offered a referral for (muscle-strengthening) exercises and & 423 & $211(49.9)$ \\
exercise activities & & \\
Advised to lose weight & 303 & $111(36.6)$ \\
Was offered a referral to services for losing weight & 247 & $37(15.0)$ \\
Assessment of problems in daily activities & 301 & $89(29.6)$ \\
Assessed for walking aid & 273 & $65(23.8)$ \\
Assessed for daily living appliances/aids & 284 & $52(18.3)$ \\
Assessment of pain & 426 & $240(56.3)$ \\
Advised paracetamol & 424 & $312(73.6)$ \\
Offered stronger painkillers & 421 & $132(31.4)$ \\
Offered anti-inflammatory medicine & 422 & $229(54.3)$ \\
Offered joint injection & 429 & $239(55.7)$ \\
Conversation about knee replacement surgery & 362 & $223(61.6)$ \\
Discussed follow-up appointment to monitor OA and evaluate & 427 & $144(33.7)$ \\
treatment & &
\end{tabular}

*Excluding "not applicable" and "do not remember"

Conclusion: There is room for improvement from the patients' perspective to increase the quality of knee OA care in the Netherlands. References:

[1] Østerås N, et al. Arthritis Care Res (Hoboken). 2013 Jul;65(7):1043-51.

[2] Østerås N, et al. RMD Open. 2015 Oct 20;1(1):e000136.

[3] Blackburn S, Res Involv Engagem. 2016 Mar 17;2:5.

Disclosure of Interests: None declared

DOI: 10.1136/annrheumdis-2020-eular.3188

\section{FRI0646-HPR MAPPING THE PATIENT JOURNEY OF NON- RADIOGRAPHIC AXIAL SPONDYLOARTHRITIS: PERSPECTIVE OF PROFESSIONALS AND PATIENTS}

T. Oton ${ }^{1}$, C. Sastré ${ }^{2}$, L. Carmona ${ }^{1} .{ }^{1}$ InMusc Instituto de Salud

Musculoesquelética, Madrid, Spain; ${ }^{2}$ Novartis Farmacéutica, S.A., Barcelona, Spain

Background: Non-radiographic axial spondyloarthritis (nr-ax-SpA) is a relatively new disease classification that has generated controversy among payers.

Objectives: To explore the perspective of patients and health care professionals (HCPs) on the journey from first symptoms to a diagnosis of non-radiographic axial spondyloarthritis (nr-ax-SpA), in order to identify gaps and unmet needs.

Methods: Qualitative study in two phases: (1) focus groups with HCPs and personal interviews with patients; (2) nominal group in which results were discussed with all stakeholders and possible solutions were proposed. Content analysis, patient journey mapping, and ideas generation techniques were used.

Results: Five focus groups were organised with rheumatologists, GP, orthopaedic surgeons, physiotherapists, and radiologists, and six patient interviews were held. HCPs recognised poor communication among specialists and contradictory or redundant approaches. Non-rheumatologists recognise poor training on spondyloarthritis, difficulty in identifying red-flags, and biases in differential diagnosis. Rheumatologists recognise that SpA nomenclature can be confusing, nevertheless nr-ax-SpA term is defining an early stage of ankylosing spondylitis, and it could lead to over-diagnosis.

Most of the patients agreed in the narrative of a very long journey with a multitude of diagnoses, mostly wrong, ineffective treatments and much frustration; acknowledging the need for psychological support during the process and the importance of receiving a diagnosis in order to cope with the disease.

The participants in the nominal group meeting recognised and discussed the problems derived from the diagnostic delay and care gaps that clearly affect people with nr-ax-SpA (Table 1).
Table 1. Problems recognised by the different actors involved in the journey.

\begin{tabular}{|c|c|c|c|c|c|c|}
\hline \multirow[t]{2}{*}{ Problem } & \multicolumn{6}{|c|}{ Recognised by } \\
\hline & Pt & PT & Or & GP & $\mathbf{R h}$ & Ra \\
\hline Non-recognition of symptoms & $\mathrm{X}$ & $\mathrm{X}$ & & & & \\
\hline Delay first visit to doctor & $\mathrm{x}$ & & & & & \\
\hline Cancel appointments & $x$ & & & & & \\
\hline Self-medication & $\mathrm{x}$ & & & & & \\
\hline Disease denial & $\mathrm{X}$ & & & & & \\
\hline Invisibility & $\mathrm{x}$ & & & & & \\
\hline Lack of adherence & $\mathrm{x}$ & & & & & \\
\hline Ignorance of medication & $\mathrm{x}$ & & & & & \\
\hline Unclear symptoms & $x$ & $\mathrm{x}$ & $\mathrm{x}$ & $\mathrm{x}$ & & \\
\hline No clinical filter by back problems & & $\mathrm{x}$ & & & & \\
\hline Treatments variability & & $\mathrm{x}$ & & & & \\
\hline Ineffective protocols & & $x$ & $x$ & & & \\
\hline Lack of time & & $\mathrm{x}$ & $\mathrm{x}$ & $\mathrm{x}$ & $\mathrm{x}$ & \\
\hline Lack of resources (human and material) & & $\mathrm{x}$ & $\mathrm{x}$ & $\mathrm{x}$ & & \\
\hline Lack of commitment & & $x$ & $\mathrm{x}$ & $\mathrm{x}$ & $\mathrm{x}$ & $\mathrm{x}$ \\
\hline Not outcome measurement & & $\mathrm{x}$ & $\mathrm{x}$ & $\mathrm{x}$ & $\mathrm{x}$ & \\
\hline Inadequate knowledge/training & & $\mathrm{x}$ & $\mathrm{x}$ & $\mathrm{x}$ & & \\
\hline Few explanations / patient education & & $x$ & $\mathrm{x}$ & $\mathrm{x}$ & $\mathrm{x}$ & \\
\hline Focused on "our" diagnostic codes & & $x$ & $x$ & $x$ & $x$ & $x$ \\
\hline No relation between specialties & & $\mathrm{x}$ & $\mathrm{x}$ & $\mathrm{x}$ & $\mathrm{x}$ & $\mathrm{x}$ \\
\hline Prioritization of tests over solutions & & $x$ & & $x$ & & $\mathrm{x}$ \\
\hline Labelling patients (prejudice) & & $\mathrm{x}$ & $\mathrm{x}$ & $\mathrm{x}$ & $\mathrm{x}$ & \\
\hline Inadequate referral circuits & & $\mathrm{x}$ & $\mathrm{x}$ & $\mathrm{x}$ & $\mathrm{x}$ & $\mathrm{x}$ \\
\hline Demotivating delays & & & $\mathrm{x}$ & & & \\
\hline Limited medical history / little research & & & $\mathrm{x}$ & & & \\
\hline Temporality of work contracts & & & & $\mathrm{x}$ & & \\
\hline Limited access to tests & & $\mathrm{x}$ & & $\mathrm{x}$ & & \\
\hline Non-sustainability threat & & & & $\mathrm{x}$ & & \\
\hline Lack of specialised support & & & & $\mathrm{x}$ & & \\
\hline Diagnostic dispersion & & & & $\mathrm{x}$ & & \\
\hline Too much weight of local issues & & & & $\mathrm{x}$ & & \\
\hline Absence of protocols or outdated & & $\mathrm{x}$ & & $\mathrm{x}$ & & \\
\hline Poor information transmission in all directions & $\mathrm{x}$ & $\mathrm{x}$ & $\mathrm{x}$ & $\mathrm{x}$ & $\mathrm{x}$ & $\mathrm{x}$ \\
\hline Focus on pharmacological treatments & & & & & $x$ & \\
\hline Ignorance of others' roles & & & & & $x$ & \\
\hline Gender bias & & & & & $\mathrm{x}$ & \\
\hline Incomplete order forms & & & & & & $\mathrm{x}$ \\
\hline
\end{tabular}

Abbreviations: Pt, patient; PT, physical therapist; Or, orthopaedic surgeon; GP, general practitioner; Rh, rheumatologist; $\mathrm{Ra}$, radiologist.

The following were indicated as possible solutions: (1) Improving relations between specialities, (2) High resolution consultations, (3) Rethinking disability scales, (4) Better information, (5) Visibility, (6) Resource maps and (7) Citizen training.

Conclusion: The patient's journey with an nr-ax-SpA is long, complicated and frustrating for both the person who experiences it and the HCPs who care for them. It is necessary to improve the knowledge about nr-ax-SpA among non-rheumatology health HCPs along with low back pain in general, among doctors and the general population, as well as other feasible measures that affect multiple levels.

Disclosure of Interests: Teresa Oton Consultant of: Novartis Farmaceutica, SA Pfizer, S.L.U., Merck Sharp \& Dohme España, S.A., Roche Farma, S.A, Sanof Aventis, AbbVie Spain, S.L.U., and Laboratorios Gebro Pharma, SA (All trhough institution), Carlos Sastré Employee of: YES; I'm Medical Advisor in Novartis Spain, Loreto Carmona Grant/research support from: Novartis Farmaceutica, SA, Pfizer, S.L.U., Merck Sharp \& Dohme España, S.A., Roche Farma, S.A, Sanofi Aventis, AbbVie Spain, S.L.U., and Laboratorios Gebro Pharma, SA (All trhough institution)

DOI: 10.1136/annrheumdis-2020-eular.104

\section{FRIDAY, 05 JUNE 2020}

\section{Rehabilitation}

\begin{tabular}{|l|l}
\hline FRI0647 & COMPARATIVE EFFECTIVENESS OF LAND AND \\
WATER-BASED EXERCISE PROGRAMS ON FATIGUE \\
IN WOMEN WITH FIBROMYALGIA: PRELIMINARY \\
FINDINGS FROM THE AL-ÁNDALUS RANDOMISED \\
CONTROLLED TRIAL.
\end{tabular}

B. Gavilán Carrera ${ }^{1,2}$, V. Segura-Jiménez ${ }^{3}$, P. Acosta-Manzano ${ }^{1,2}$, M. Borges Cosic $^{1,2}$, F. Estévez-López ${ }^{4}$, M. Delgado-Fernández ${ }^{1,2} .{ }^{1}$ Faculty of Sport Sciences, University of Granada, Department of Physical Education and Sports, Granada, Spain; ${ }^{2}$ Sport and Health University Research Institute (iMUDS), Granada, Spain; ${ }^{3}$ Faculty of Education Sciences, University of Cádiz, Department of Physical Education, Puerto Real, Spain; ${ }^{4}$ Erasmus MC University 